\title{
Transitivity of generalized intuitionistic fuzzy matrices
}

Riyaz Ahmad Padder and P. Murugadas

Department of Mathematics, Annamalai University, India

Received: 18 August 2016, Accepted: 25 November 2016

Published online: 13 November 2017.

\begin{abstract}
In this paper, generalized intuitionistic fuzzy matrices are considered as matrices over a special type of semiring which is called path algebra. We introduce the concept of transitivity of generalized intuitionistic fuzzy matrices. Some algebraic properties of generalized intuitionistic fuzzy matrices are developed. Also, we develop some properties of transitivity.
\end{abstract}

Keywords: Intuitionistic fuzzy sets, Intuitionistic fuzzy matrix, Transitivity, Generalized intuitionistic fuzzy matrix.

\section{Introduction}

In real life scenario, we frequently deal with the information which is some times vague, some times inexact or imprecise and occasionally insufficient. Zadeh's classical concept of fuzzy sets [1] is strong enough to deal with such type of problems. In fuzzy set theory, the membership of an element to a fuzzy set is a single value between zero and one. However in reality, it may not be always true that the degree of non-membership of an element in a fuzzy set is equal to 1 minus the membership degree because there may be some hesitation degree. To overcome, these difficulties, Atanassov $[2,3]$ developed the theory of intuitionistic fuzzy sets (IFSs) as a generalization of fuzzy sets. Lot of research works were done by several researchers on the field of IFSs.

Matrices play a vital role in various areas of science and Engineering. The classical matrix theory can not solve the problems involving various types of uncertainties. That type of problems are solved by using fuzzy matrix (FM) [4]. Kim and Roush [5] developed the concept of Generalized Fuzzy Matrices (GFMs). Transitive matrices are an important type of generalized matrices which represent transitive relation $[6,7,8,9,10]$. Transitive relation plays an important role in clustering, information retrieval, preference, and so on $[9,11,12]$. The transitivity problems of matrices over some special semirings have been discussed by many authors [13,14,15,16,17,18,19,20]. Hashimoto [15] presented the concept of transitive FMs and considered the convergence of powers of transitive FMs. Hashimoto [16] studied the canonical form of a transitive FM. Xin [21,22] studied controllable FMs. Koodziejczy [17] gave the concept of s-transitive FMs and considered the convergence of powers of s-transitive FMs. Tan [19,20] discussed the convergence of powers of transitive lattice matrices. Jiang [23] studied the transitive incline matrices. Some elementary properties and characterizations for transitive GFMs are established and transitivity of powers of a GFM were discussed [24]. Pal [25] introduced intuitionistic fuzzy determinant. Pal, et al., [26] studied intuitionistic fuzzy matrices (IFMs). Khan and Pal [27] studied intuitionistic fuzzy tautological matrices and also studied interval-valued IFMs [28]. Bhowmik and Pal [29, 30] introduced some results on IFMs and intuitionistic circulant FMs and GIFMs. Khan and Pal [31] introduced the concept of generalized inverse for IFMs. Hong and Nae [32] studied some properties of canonical form of transitive IFM. Some algebraic properties of GIFMs are presented over distributive lattice [33]. Some results are investigated regarding the group inverse of IFMs [34]. Several authors [35,36,37,38,39,40,41,42,43,44,45,46,47,48] worked on 
IFMs and obtained various interesting results which are very useful in handling uncertainty problems in our daily life. An interesting problem in the theory of IFM is the transitivity of GIFM. Many authors worked on this problem.

\section{Definitions}

Definition 1. [2] An Intuitionistic Fuzzy Set (IFS) A in X (universal set) is defined as an object of the following form $A=\left\{\left\langle x, \mu_{A}(x), v_{A}(x)\right\rangle / x \in X\right\}$, where the functions: $\mu_{A}: X \rightarrow[0,1]$ and $v_{A}: X \rightarrow[0,1]$ define the membership function and non-membership function of the element $x \in X$ respectively and for every $x \in X: 0 \leq \mu_{A}(x)+v_{A}(x) \leq 1$.

In short we write $\left\langle x, x^{\prime}\right\rangle$ as an intuitionistic fuzzy element with $x+x^{\prime} \leq$. For $\left\langle x, x^{\prime}\right\rangle,\left\langle y, y^{\prime}\right\rangle \in$ IFS, Atanassov introduced operations $\left\langle x, x^{\prime}\right\rangle \vee\left\langle y, y^{\prime}\right\rangle=\left\langle\max \{x, y\}, \min \left\{x^{\prime}, y^{\prime}\right\}\right\rangle,\left\langle x, x^{\prime}\right\rangle \wedge\left\langle y, y^{\prime}\right\rangle=\left\langle\min \{x, y\}, \max \left\{x^{\prime}, y^{\prime}\right\}\right\rangle$, if $\left\langle x, x^{\prime}\right\rangle \leq\left\langle y, y^{\prime}\right\rangle$ means $x \leq y, x^{\prime} \geq y^{\prime}$ and $\left\langle x, x^{\prime}\right\rangle\left\langle\left\langle y, y^{\prime}\right\rangle\right.$ if $x<y$ and $x^{\prime}>y^{\prime}$; in this case we say $\left\langle x, x^{\prime}\right\rangle,\left\langle y, y^{\prime}\right\rangle$ are comparable. For any two comparable elements $\left\langle x, x^{\prime}\right\rangle,\left\langle y, y^{\prime}\right\rangle \in$ IFS, the operations $\left\langle x, x^{\prime}\right\rangle-\left\langle y, y^{\prime}\right\rangle$ and $\left\langle x, x^{\prime}\right\rangle \leftarrow\left\langle y, y^{\prime}\right\rangle$ are defined by

$$
\begin{aligned}
& \left\langle x, x^{\prime}\right\rangle-\left\langle y, y^{\prime}\right\rangle= \begin{cases}\left\langle x, x^{\prime}\right\rangle & \text { if }\left\langle x, x^{\prime}\right\rangle>\left\langle y, y^{\prime}\right\rangle, \\
\langle 0,1\rangle & \text { if }\left\langle x, x^{\prime}\right\rangle \leq\left\langle y, y^{\prime}\right\rangle .\end{cases} \\
& \left\langle x, x^{\prime}\right\rangle \leftarrow\left\langle y, y^{\prime}\right\rangle= \begin{cases}\langle 1,0\rangle & \text { if }\left\langle x, x^{\prime}\right\rangle \geq\left\langle y, y^{\prime}\right\rangle, \\
\left\langle x, x^{\prime}\right\rangle & \text { if }\left\langle x, x^{\prime}\right\rangle<\left\langle y, y^{\prime}\right\rangle .\end{cases}
\end{aligned}
$$

Definition 2. [26] Let $X=\left\{x_{1}, x_{2}, \ldots x_{m}\right\}$ be a set of alternatives and $Y=\left\{y_{1}, y_{2}, \ldots y_{n}\right\}$ be the attribute set of each element of $X$. An IFM is defined by $A=\left(\left\langle\left(x_{i}, y_{j}\right), \mu_{A}\left(x_{i}, y_{j}\right), v_{A}\left(x_{i}, y_{j}\right)\right\rangle\right)$ for $i=1,2 \ldots$ m and $j=1,2, \ldots n$, where $\mu_{A}: X \times Y \rightarrow[0,1]$ and $v_{A}: X \times Y \rightarrow[0,1]$ satisfy the condition $0 \leq \mu_{A}\left(x_{i}, y_{j}\right)+v_{A}\left(x_{i}, y_{j}\right) \leq 1$. For simplicity we denote an IFM is a matrix of pairs $A=\left(\left\langle a_{i j}, a_{i j}^{\prime}\right\rangle\right)$ of non negative real numbers satisfying $a_{i j}+a_{i j}^{\prime} \leq 1$ for all $i, j$. We denote the set of all IFM of order $m \times n$ by $\mathscr{F}_{m n}$ and $\mathscr{F}_{n}$ denotes the set of IFM of order $n \times n$.

Definition 3. For intuitionistic fuzzy matrices $A=\left(\left\langle a_{i j}, a_{i j}^{\prime}\right\rangle\right), B=\left(\left\langle b_{i j}, b_{i j}^{\prime}\right\rangle\right), C=\left(\left\langle c_{i j}, c_{i j}^{\prime}\right\rangle\right) \in \mathscr{F}_{m n}$.

Some matrix operations are given below

$$
\begin{aligned}
& A \wedge B=\left(\left\langle a_{i j} \wedge b_{i j}, a_{i j}^{\prime} \vee b_{i j}^{\prime}\right\rangle\right) \\
& A \times C=\left(\bigvee_{k=1}^{n}\left(\left(\left\langle a_{i k}, a_{i k}^{\prime}\right\rangle\right) \wedge\left(\left\langle c_{k j}, c_{k j}^{\prime}\right\rangle\right)\right)\right) \\
& A \stackrel{c}{\leftarrow} B=\left(\left\langle a_{i j}, a_{i j}^{\prime}\right\rangle \stackrel{c}{\leftarrow}\left\langle b_{i j}, b_{i j}^{\prime}\right\rangle\right)
\end{aligned}
$$

Definition 4. For IFMs $A=\left(\left\langle a_{i j}, a_{i j}^{\prime}\right)_{(m \times l)}\right.$ and $C=\left(\left\langle c_{i j}, c_{i j}^{\prime}\right)_{(n \times l)}\right.$ over $X, A \leftarrow C$ is defined as follows

$$
A \leftarrow C=\left(\bigwedge_{k=1}^{n}\left(\left\langle a_{i k}, a_{i k}^{\prime}\right\rangle \leftarrow\left\langle c_{k j}, c_{k j}^{\prime}\right\rangle\right)\right)_{(m \times l)} .
$$

Definition 5. An intuitionistic fuzzy algebra (IFA) is a mathematical system $(A, \vee, \wedge)$ with two binary operations $\vee$, $\wedge$ defined on a set A satisfying the following properties.

(1) Idempotence $\left\langle a, a^{\prime}\right\rangle \vee\left\langle a, a^{\prime}\right\rangle=\left\langle a, a^{\prime}\right\rangle,\left\langle a, a^{\prime}\right\rangle \wedge\left\langle a, a^{\prime}\right\rangle=\left\langle a, a^{\prime}\right\rangle$ 
(2) Commutativity $\left\langle a, a^{\prime}\right\rangle \vee\left\langle b, b^{\prime}\right\rangle=\left\langle b, b^{\prime}\right\rangle \vee\left\langle a, a^{\prime}\right\rangle,\left\langle a, a^{\prime}\right\rangle \wedge\left\langle b, b^{\prime}\right\rangle=\left\langle b, b^{\prime}\right\rangle \wedge\left\langle a, a^{\prime}\right\rangle$

(3) Associativity $\left\langle a, a^{\prime}\right\rangle \vee\left(\left\langle b, b^{\prime}\right\rangle \vee\left\langle c, c^{\prime}\right\rangle\right)=\left(\left\langle a, a^{\prime}\right\rangle \vee\left\langle b, b^{\prime}\right\rangle\right) \vee\left\langle c, c^{\prime}\right\rangle$, $\left\langle a, a^{\prime}\right\rangle \wedge\left(\left\langle b, b^{\prime}\right\rangle \wedge\left\langle c, c^{\prime}\right\rangle\right)=\left(\left\langle a, a^{\prime}\right\rangle \wedge\left\langle b, b^{\prime}\right\rangle\right) \wedge\left\langle c, c^{\prime}\right\rangle$

(4) Absorption $\left\langle a, a^{\prime}\right\rangle \vee\left(\left\langle a, a^{\prime}\right\rangle \wedge\left\langle b, b^{\prime}\right\rangle\right)=\left\langle a, a^{\prime}\right\rangle,\left\langle a, a^{\prime}\right\rangle \wedge\left(\left\langle a, a^{\prime}\right\rangle \vee\left\langle b, b^{\prime}\right\rangle\right)=\left\langle a, a^{\prime}\right\rangle$

(5) Distributivity $\left\langle a, a^{\prime}\right\rangle \vee\left(\left\langle a, a^{\prime}\right\rangle \wedge\left\langle c, c^{\prime}\right\rangle\right)=\left(\left\langle a, a^{\prime}\right\rangle \vee\left\langle b, b^{\prime}\right\rangle\right) \wedge\left(\left\langle a, a^{\prime}\right\rangle \vee\left\langle c, c^{\prime}\right\rangle\right)$. $\left\langle a, a^{\prime}\right\rangle \wedge\left(\left\langle a, a^{\prime}\right\rangle \vee\left\langle c, c^{\prime}\right\rangle\right)=\left(\left\langle a, a^{\prime}\right\rangle \wedge\left\langle b, b^{\prime}\right\rangle\right) \vee\left(\left\langle a, a^{\prime}\right\rangle \wedge\left\langle c, c^{\prime}\right\rangle\right)$.

(6) Universal bounds

$\left\langle a, a^{\prime}\right\rangle \vee\langle 0,1\rangle=\left\langle a, a^{\prime}\right\rangle,\left\langle a, a^{\prime}\right\rangle \vee\langle 1,0\rangle=\langle 1,0\rangle,\left\langle a, a^{\prime}\right\rangle \wedge\langle 0,1\rangle=\langle 0,1\rangle,\left\langle a, a^{\prime}\right\rangle \wedge\langle 1,0\rangle=\left\langle a, a^{\prime}\right\rangle$

\section{Results}

In the IFA, we omit some of the condition and we define the following and study some important results using this new structure.

Definition 6. Let $X$ be a set and a few $\langle 0,1\rangle,\langle 1,0\rangle$ and all $\left\langle x, x^{\prime}\right\rangle,\left\langle y, y^{\prime}\right\rangle,\left\langle z, z^{\prime}\right\rangle \in X$, operations $\wedge$ and $\vee$ satisfy the following properties

$$
\begin{aligned}
& \left\langle x, x^{\prime}\right\rangle \vee\left\langle y, y^{\prime}\right\rangle \in X, \quad\left\langle x, x^{\prime}\right\rangle \wedge\left\langle y, y^{\prime}\right\rangle \in X \\
& \left\langle x, x^{\prime}\right\rangle \vee\left\langle y, y^{\prime}\right\rangle=\left\langle y, y^{\prime}\right\rangle \vee\left\langle x, x^{\prime}\right\rangle \\
& \left(\left\langle x, x^{\prime}\right\rangle \vee\left\langle y, y^{\prime}\right\rangle\right) \vee\left\langle z, z^{\prime}\right\rangle=\left\langle x, x^{\prime}\right\rangle \vee\left(\left\langle y, y^{\prime}\right\rangle \vee\left\langle z, z^{\prime}\right\rangle\right) \\
& \left(\left\langle x, x^{\prime}\right\rangle \wedge\left\langle y, y^{\prime}\right\rangle\right) \wedge\left\langle z, z^{\prime}\right\rangle=\left\langle x, x^{\prime}\right\rangle \wedge\left(\left\langle y, y^{\prime}\right\rangle \wedge\left\langle z, z^{\prime}\right\rangle\right) \\
& \left\langle x, x^{\prime}\right\rangle \wedge\left(\left\langle y, y^{\prime}\right\rangle \vee\left\langle z, z^{\prime}\right\rangle\right)=\left(\left\langle x, x^{\prime}\right\rangle \wedge\left\langle y, y^{\prime}\right\rangle\right) \vee\left(\left\langle x, x^{\prime}\right\rangle \wedge\left\langle z, z^{\prime}\right\rangle\right), \\
& \left(\left\langle x, x^{\prime}\right\rangle \vee\left\langle y, y^{\prime}\right\rangle\right) \wedge\left\langle z, z^{\prime}\right\rangle=\left(\left\langle x, x^{\prime}\right\rangle \wedge\left\langle z, z^{\prime}\right\rangle\right) \vee\left(\left\langle y, y^{\prime}\right\rangle \wedge\left\langle z, z^{\prime}\right\rangle\right), \\
& \left\langle x, x^{\prime}\right\rangle \vee\left\langle x, x^{\prime}\right\rangle=\left\langle x, x^{\prime}\right\rangle \\
& \left\langle x, x^{\prime}\right\rangle \vee\langle 0,1\rangle=\left\langle x, x^{\prime}\right\rangle \\
& \left\langle x, x^{\prime}\right\rangle \wedge\langle 0,1\rangle=\langle 0,1\rangle \wedge\left\langle x, x^{\prime}\right\rangle=\langle 0,1\rangle \\
& \left\langle x, x^{\prime}\right\rangle \wedge\langle 1,0\rangle=\langle 1,0\rangle \wedge\left\langle x, x^{\prime}\right\rangle=\left\langle x, x^{\prime}\right\rangle
\end{aligned}
$$

Note 1.The Definition 6, does not contain $\left\langle x, x^{\prime}\right\rangle \wedge\left\langle y, y^{\prime}\right\rangle=\left\langle y, y^{\prime}\right\rangle \wedge\left\langle x, x^{\prime}\right\rangle,\left\langle x, x^{\prime}\right\rangle \wedge\left\langle x, x^{\prime}\right\rangle=\left\langle x, x^{\prime}\right\rangle$ and $\left\langle x, x^{\prime}\right\rangle \vee\langle 1,0\rangle=\langle 1,0\rangle$

Definition 7. For every $\left\langle x, x^{\prime}\right\rangle,\left\langle y, y^{\prime}\right\rangle \in X,\left\langle x, x^{\prime}\right\rangle \preceq\left\langle y, y^{\prime}\right\rangle$ if and only if $\left\langle x, x^{\prime}\right\rangle \vee\left\langle y, y^{\prime}\right\rangle=\left\langle y, y^{\prime}\right\rangle$. Here it is not necessary that $\left\langle x, x^{\prime}\right\rangle \wedge\left\langle y, y^{\prime}\right\rangle=\left\langle x, x^{\prime}\right\rangle$.

Definition 8. $A \preceq B$ if and only if $\left\langle a_{i j}, a_{i j}^{\prime}\right\rangle \preceq\left\langle a_{i j}, a_{i j}^{\prime}\right\rangle$ for all $i, j$.

Remark. Let $Q$ be IFM $n \times n$ over $X$, then $Q$ is transitive iff $Q^{2}=Q \times Q \preceq Q$.

Remark. Let $Q=\left(\left\langle q_{i j}, q_{i j}^{\prime}\right\rangle\right)$ be an $n \times n$ IFM over $X$ is transitive if and only if $\left\langle q_{i k}, q_{i k}^{\prime}\right\rangle \wedge\left\langle q_{k j}, q_{k j}^{\prime}\right\rangle \preceq\left\langle q_{i j}, q_{i j}^{\prime}\right\rangle$ for all $k$.

Remark. An IFM which satisfies the conditions of Definition 6, only is called GIFM. 
Lemma 1. The following results are trivial from Definition 6. Let $\left\langle x, x^{\prime}\right\rangle,\left\langle y, y^{\prime}\right\rangle,\left\langle z, z^{\prime}\right\rangle,\left\langle u, u^{\prime}\right\rangle,\left\langle v, v^{\prime}\right\rangle \in X$,

(a) if $\left\langle x, x^{\prime}\right\rangle \preceq\left\langle y, y^{\prime}\right\rangle,\left\langle y, y^{\prime}\right\rangle \preceq\left\langle z, z^{\prime}\right\rangle$, then $\left\langle x, x^{\prime}\right\rangle \preceq\left\langle z, z^{\prime}\right\rangle$,

(b) if $\left\langle x, x^{\prime}\right\rangle \preceq\left\langle y, y^{\prime}\right\rangle,\left\langle u, u^{\prime}\right\rangle \preceq\left\langle v, v^{\prime}\right\rangle$, then $\left\langle x, x^{\prime}\right\rangle \vee\left\langle u, u^{\prime}\right\rangle \preceq\left\langle y, y^{\prime}\right\rangle \vee\left\langle v, v^{\prime}\right\rangle,\left\langle x, x^{\prime}\right\rangle \wedge\left\langle u, u^{\prime}\right\rangle \preceq\left\langle y, y^{\prime}\right\rangle \wedge\left\langle v, v^{\prime}\right\rangle$,

(c) if $\left\langle x, x^{\prime}\right\rangle \preceq\left\langle y, y^{\prime}\right\rangle,\left\langle y, y^{\prime}\right\rangle \preceq\left\langle x, x^{\prime}\right\rangle$, then $\left\langle x, x^{\prime}\right\rangle=\left\langle y, y^{\prime}\right\rangle$,

Theorem 1. Let $\left\langle x, x^{\prime}\right\rangle *\left\langle y, y^{\prime}\right\rangle \in X$ be an operation such that

$$
\left(\left\langle x, x^{\prime}\right\rangle *\left\langle y, y^{\prime}\right\rangle\right) \wedge\left(\left\langle u, u^{\prime}\right\rangle *\left\langle v, v^{\prime}\right\rangle\right) \preceq\left(\left\langle x, x^{\prime}\right\rangle \wedge\left\langle u, u^{\prime}\right\rangle\right) *\left(\left\langle y, y^{\prime}\right\rangle \wedge\left\langle v, v^{\prime}\right\rangle\right)
$$

and if $\left\langle x, x^{\prime}\right\rangle \preceq\left\langle y, y^{\prime}\right\rangle,\left\langle u, u^{\prime}\right\rangle \preceq\left\langle v, v^{\prime}\right\rangle$, then $\left\langle x, x^{\prime}\right\rangle *\left\langle u, u^{\prime}\right\rangle \preceq\left\langle y, y^{\prime}\right\rangle *\left\langle v, v^{\prime}\right\rangle$ for every $\left\langle x, x^{\prime}\right\rangle,\left\langle y, y^{\prime}\right\rangle,\left\langle u, u^{\prime}\right\rangle,\left\langle v, v^{\prime}\right\rangle \in X$. If $Q$ and $S$ are transitive then $Q * S$, is transitive, where $Q * S$ is defined component-wise.

Proof. Since $Q$ and $S$ are transitive, we have

$$
\left\langle q_{i k}, q_{i k}^{\prime}\right\rangle \wedge\left\langle q_{k j}, q_{k j}^{\prime}\right\rangle \preceq\left\langle q_{i j}, q_{i j}^{\prime}\right\rangle,\left\langle s_{i k}, s_{i k}^{\prime}\right\rangle \wedge\left\langle s_{k j}, s_{k j}^{\prime}\right\rangle \preceq\left\langle s_{i j}, s_{i j}^{\prime}\right\rangle
$$

By applying the properties of $*$,

$$
\left(\left\langle q_{i k}, q_{i k}^{\prime}\right\rangle \wedge\left\langle q_{k j}, q_{k j}^{\prime}\right\rangle\right) *\left(\left\langle s_{i k}, s_{i k}^{\prime}\right\rangle \wedge\left\langle s_{k j}, s_{k j}^{\prime}\right\rangle\right) \preceq\left\langle q_{i j}, q_{i j}^{\prime}\right\rangle *\left\langle s_{i j}, s_{i j}^{\prime}\right\rangle
$$

so that

$$
\left(\left\langle q_{i k}, q_{i k}^{\prime}\right\rangle \wedge\left\langle s_{i k}, s_{i k}^{\prime}\right\rangle\right) *\left(\left\langle q_{k j}, q_{k j}^{\prime}\right\rangle \wedge\left\langle s_{k j}, s_{k j}^{\prime}\right\rangle\right) \preceq\left\langle q_{i j}, q_{i j}^{\prime}\right\rangle *\left\langle s_{i j}, s_{i j}^{\prime}\right\rangle
$$

From Remark $2 ; Q * S$ is transitive.

Example 1. $X=\left\{\left\langle x, x^{\prime}\right\rangle \mid\langle 0,1\rangle \leq\left\langle x, x^{\prime}\right\rangle \leq\langle 1,0\rangle\right\}$,

$$
\begin{aligned}
& \left\langle x, x^{\prime}\right\rangle \vee\left\langle y, y^{\prime}\right\rangle=\left\langle\min \{x, y\}, \max \left\{x^{\prime}, y^{\prime}\right\}\right\rangle \\
& \left\langle x, x^{\prime}\right\rangle \wedge\left\langle y, y^{\prime}\right\rangle=\left\langle\min \{x+y, 1\}, \max \left\{1-\left(x^{\prime}+y^{\prime}\right), 0\right\}\right\rangle \\
& \left\langle x, x^{\prime}\right\rangle *\left\langle y, y^{\prime}\right\rangle=\left\langle\max \{x, y\}, \min \left\{x^{\prime}, y^{\prime}\right\}\right\rangle \text { for every }\left\langle x, x^{\prime}\right\rangle,\left\langle y, y^{\prime}\right\rangle \in X . \quad \text { Then } \\
& \left\langle x, x^{\prime}\right\rangle \preceq\left\langle y, y^{\prime}\right\rangle \quad \text { if and only if }\left\langle x, x^{\prime}\right\rangle \geq\left\langle y, y^{\prime}\right\rangle \\
& \left(\left\langle x, x^{\prime}\right\rangle *\left\langle y, y^{\prime}\right\rangle\right) \wedge\left(\left\langle u, u^{\prime}\right\rangle *\left\langle v, v^{\prime}\right\rangle\right) \preceq\left(\left\langle x, x^{\prime}\right\rangle \wedge\left\langle u, u^{\prime}\right\rangle\right) *\left(\left\langle y, y^{\prime}\right\rangle \wedge\left\langle v, v^{\prime}\right\rangle\right)
\end{aligned}
$$

Corollary 1. Let $Q$ and $S$ be $n \times n$ transitive IFMs, $\wedge$ is commutative, then $Q \wedge S$ is transitive.

Corollary 2. Let $f\left(\left\langle x, x^{\prime}\right\rangle\right) \in X$ be a function such that

$$
f\left(\left\langle x, x^{\prime}\right\rangle\right) \wedge f\left(\left\langle y, y^{\prime}\right\rangle\right) \preceq f\left(\left\langle x, x^{\prime}\right\rangle \wedge\left\langle y, y^{\prime}\right\rangle\right)
$$

and if $\left\langle x, x^{\prime}\right\rangle \preceq\left\langle y, y^{\prime}\right\rangle$ then $f\left(\left\langle x, x^{\prime}\right\rangle\right) \preceq f\left(\left\langle y, y^{\prime}\right\rangle\right)$ for all $\left\langle x, x^{\prime}\right\rangle,\left\langle y, y^{\prime}\right\rangle \in X$. If $Q$ is transitive, then $f(Q)$ is transitive, where $f(Q)=\left[f\left(\left\langle q_{i j}, q_{i j}^{\prime}\right\rangle\right)\right]$

Example 2. Let $X=\left\{\left\langle x, x^{\prime}\right\rangle \mid\langle 0,1\rangle \leq\left\langle x, x^{\prime}\right\rangle \leq\langle 1,0\rangle\right\}$.

Let $\left\langle x, x^{\prime}\right\rangle \vee\left\langle y, y^{\prime}\right\rangle=\left\langle\max \{x, y\}, \min \left\{x^{\prime}, y^{\prime}\right\}\right\rangle$ and $\left\langle x, x^{\prime}\right\rangle \wedge\left\langle y, y^{\prime}\right\rangle=\left\langle x, x^{\prime}\right\rangle\left\langle y, y^{\prime}\right\rangle=\left\langle x y, x^{\prime} y^{\prime}\right\rangle$ $f\left(\left\langle x, x^{\prime}\right\rangle\right)=\left\langle c, c^{\prime}\right\rangle\left\langle x, x^{\prime}\right\rangle=\left\langle c x, c^{\prime} x^{\prime}\right\rangle,\left\langle c, c^{\prime}\right\rangle \in X$ for all $\left\langle x, x^{\prime}\right\rangle,\left\langle y, y^{\prime}\right\rangle \in X . \Rightarrow\left\langle x, x^{\prime}\right\rangle \preceq\left\langle y, y^{\prime}\right\rangle$ iff $\left\langle x, x^{\prime}\right\rangle \leq\left\langle y, y^{\prime}\right\rangle$ $f\left(\left\langle x, x^{\prime}\right\rangle\right) \wedge f\left(\left\langle y, y^{\prime}\right\rangle\right)=\left\langle c, c^{\prime}\right\rangle\left\langle x, x^{\prime}\right\rangle\left\langle c, c^{\prime}\right\rangle\left\langle y, y^{\prime}\right\rangle=\left\langle c^{2}, c^{\prime 2}\right\rangle\left\langle x, x^{\prime}\right\rangle\left\langle y, y^{\prime}\right\rangle$, and $f\left(\left\langle x, x^{\prime}\right\rangle \wedge\left\langle y, y^{\prime}\right\rangle\right)=\left\langle c, c^{\prime}\right\rangle\left\langle x, x^{\prime}\right\rangle\left\langle y, y^{\prime}\right\rangle$. Thus $f\left(\left\langle x, x^{\prime}\right\rangle\right) \wedge f\left(\left\langle y, y^{\prime}\right\rangle\right) \preceq f\left(\left\langle x, x^{\prime}\right\rangle \wedge\left\langle y, y^{\prime}\right\rangle\right)$. 
Proposition 1. If $\left\langle x, x^{\prime}\right\rangle,\left\langle y, y^{\prime}\right\rangle,\left\langle u, u^{\prime}\right\rangle,\left\langle v, v^{\prime}\right\rangle \in X,\left\langle x, x^{\prime}\right\rangle *\left\langle x, x^{\prime}\right\rangle=\left\langle x, x^{\prime}\right\rangle, \quad\left\langle x, x^{\prime}\right\rangle *\left\langle y, y^{\prime}\right\rangle \preceq\left\langle x, x^{\prime}\right\rangle, \quad\left\langle x, x^{\prime}\right\rangle *\left\langle y, y^{\prime}\right\rangle \preceq\left\langle y, y^{\prime}\right\rangle$, and $\left\langle x, x^{\prime}\right\rangle \preceq\left\langle y, y^{\prime}\right\rangle,\left\langle u, u^{\prime}\right\rangle \preceq\left\langle v, v^{\prime}\right\rangle$, $\Rightarrow\left\langle x, x^{\prime}\right\rangle *\left\langle u, u^{\prime}\right\rangle \preceq\left\langle y, y^{\prime}\right\rangle *\left\langle v, v^{\prime}\right\rangle$, then

(a) $\left\langle x, x^{\prime}\right\rangle *\left\langle y, y^{\prime}\right\rangle=\left\langle y, y^{\prime}\right\rangle *\left\langle x, x^{\prime}\right\rangle$,

(b) $\left(\left\langle x, x^{\prime}\right\rangle *\left\langle y, y^{\prime}\right\rangle\right) \wedge\left(\left\langle u, u^{\prime}\right\rangle *\left\langle v, v^{\prime}\right\rangle\right) \preceq\left(\left\langle x, x^{\prime}\right\rangle \wedge\left\langle u, u^{\prime}\right\rangle\right) *\left(\left\langle y, y^{\prime}\right\rangle \wedge\left\langle v, v^{\prime}\right\rangle\right)$

(c) $\left\langle x, x^{\prime}\right\rangle \preceq\left\langle y, y^{\prime}\right\rangle \Rightarrow\left\langle x, x^{\prime}\right\rangle *\left\langle y, y^{\prime}\right\rangle=\left\langle x, x^{\prime}\right\rangle$

Proof. (a) By $\left\langle x, x^{\prime}\right\rangle *\left\langle y, y^{\prime}\right\rangle \preceq\left\langle y, y^{\prime}\right\rangle$, and $\left\langle x, x^{\prime}\right\rangle *\left\langle y, y^{\prime}\right\rangle \preceq\left\langle x, x^{\prime}\right\rangle$, we get

$\left(\left\langle x, x^{\prime}\right\rangle *\left\langle y, y^{\prime}\right\rangle\right) *\left(\left\langle x, x^{\prime}\right\rangle *\left\langle y, y^{\prime}\right\rangle\right) \preceq\left(\left\langle y, y^{\prime}\right\rangle *\left\langle x, x^{\prime}\right\rangle\right) \Rightarrow\left(\left\langle x, x^{\prime}\right\rangle *\left\langle y, y^{\prime}\right\rangle\right) \preceq\left(\left\langle y, y^{\prime}\right\rangle *\left\langle x, x^{\prime}\right\rangle\right)$.

Similarly we can prove $\left(\left\langle y, y^{\prime}\right\rangle *\left\langle x, x^{\prime}\right\rangle\right) \preceq\left(\left\langle x, x^{\prime}\right\rangle *\left\langle y, y^{\prime}\right\rangle\right)$. Hence $\left\langle x, x^{\prime}\right\rangle *\left\langle y, y^{\prime}\right\rangle=\left\langle y, y^{\prime}\right\rangle *\left\langle x, x^{\prime}\right\rangle$.

(b) $\left\langle x, x^{\prime}\right\rangle *\left\langle y, y^{\prime}\right\rangle \preceq\left\langle x, x^{\prime}\right\rangle$, and $\left\langle u, u^{\prime}\right\rangle *\left\langle v, v^{\prime}\right\rangle \preceq\left\langle u, u^{\prime}\right\rangle$, we get $\left(\left\langle x, x^{\prime}\right\rangle *\left\langle y, y^{\prime}\right\rangle\right) \wedge\left(\left\langle u, u^{\prime}\right\rangle *\left\langle v, v^{\prime}\right\rangle\right) \preceq\left(\left\langle x, x^{\prime}\right\rangle \wedge\left\langle u, u^{\prime}\right\rangle\right)$

Similarly we can prove that, $\left(\left\langle x, x^{\prime}\right\rangle *\left\langle y, y^{\prime}\right\rangle\right) \wedge\left(\left\langle u, u^{\prime}\right\rangle *\left\langle v, v^{\prime}\right\rangle\right) \preceq\left(\left\langle y, y^{\prime}\right\rangle \wedge\left\langle v, v^{\prime}\right\rangle\right)$

Hence $\left\langle x, x^{\prime}\right\rangle *\left\langle y, y^{\prime}\right\rangle \wedge\left\langle u, u^{\prime}\right\rangle *\left\langle v, v^{\prime}\right\rangle \preceq\left(\left\langle x, x^{\prime}\right\rangle \wedge\left\langle u, u^{\prime}\right\rangle\right) *\left(\left\langle y, y^{\prime}\right\rangle \wedge\left\langle v, v^{\prime}\right\rangle\right)$.

(c) Let $\left\langle x, x^{\prime}\right\rangle \preceq\left\langle y, y^{\prime}\right\rangle$ we get, We know that $\left\langle x, x^{\prime}\right\rangle *\left\langle x, x^{\prime}\right\rangle=\left\langle x, x^{\prime}\right\rangle$,

$\Rightarrow\left\langle x, x^{\prime}\right\rangle *\left\langle x, x^{\prime}\right\rangle \preceq\left\langle x, x^{\prime}\right\rangle *\left\langle y, y^{\prime}\right\rangle \preceq\left\langle x, x^{\prime}\right\rangle=\left\langle x, x^{\prime}\right\rangle *\left\langle x, x^{\prime}\right\rangle, \Rightarrow\left\langle x, x^{\prime}\right\rangle *\left\langle y, y^{\prime}\right\rangle=\left\langle x, x^{\prime}\right\rangle *\left\langle x, x^{\prime}\right\rangle$.

Hence $\left\langle x, x^{\prime}\right\rangle *\left\langle y, y^{\prime}\right\rangle=\left\langle x, x^{\prime}\right\rangle$.

Definition 9. An operation $\left\langle x, x^{\prime}\right\rangle-\left\langle y, y^{\prime}\right\rangle \in X$ satisfies the below condition, for every $\left\langle x, x^{\prime}\right\rangle,\left\langle y, y^{\prime}\right\rangle,\left\langle z, z^{\prime}\right\rangle \in X$, if $\left\langle x, x^{\prime}\right\rangle \preceq$ $\left\langle y, y^{\prime}\right\rangle$, then $\left\langle x, x^{\prime}\right\rangle-\left\langle y, y^{\prime}\right\rangle=\langle 0,1\rangle,\langle z, z\rangle-\left\langle y, y^{\prime}\right\rangle \preceq\left\langle z, z^{\prime}\right\rangle-\left\langle x, x^{\prime}\right\rangle,\left\langle x, x^{\prime}\right\rangle-\left\langle z, z^{\prime}\right\rangle \preceq\left\langle y, y^{\prime}\right\rangle-\left\langle z, z^{\prime}\right\rangle$

Lemma 2. If for every $\left\langle x, x^{\prime}\right\rangle,\left\langle y, y^{\prime}\right\rangle,\left\langle u, u^{\prime}\right\rangle,\left\langle v, v^{\prime}\right\rangle \in X,\left\langle x, x^{\prime}\right\rangle \preceq\left\langle y, y^{\prime}\right\rangle$ and $\left\langle u, u^{\prime}\right\rangle \preceq\left\langle v, v^{\prime}\right\rangle$, then $\left\langle x, x^{\prime}\right\rangle-\left\langle v, v^{\prime}\right\rangle \preceq\left\langle y, y^{\prime}\right\rangle-\left\langle u, u^{\prime}\right\rangle$

Proof. By the Definition 9, we get $\left\langle x, x^{\prime}\right\rangle-\left\langle v, v^{\prime}\right\rangle \preceq\left\langle x, x^{\prime}\right\rangle-\left\langle u, u^{\prime}\right\rangle \preceq\left\langle y, y^{\prime}\right\rangle-\left\langle u, u^{\prime}\right\rangle$

Lemma 3. Let $\wedge$ be idempotent and $\langle 1,0\rangle \vee\left\langle x, x^{\prime}\right\rangle=\langle 1,0\rangle$ for all $\left\langle x, x^{\prime}\right\rangle \in X$, then for every $\left\langle x, x^{\prime}\right\rangle,\left\langle y, y^{\prime}\right\rangle \in X$

(a) $\left\langle x, x^{\prime}\right\rangle \wedge\left\langle y, y^{\prime}\right\rangle \preceq\left\langle y, y^{\prime}\right\rangle$,

(b) $\left\langle x, x^{\prime}\right\rangle \wedge\left\langle y, y^{\prime}\right\rangle \preceq\left\langle x, x^{\prime}\right\rangle$

(c) $\left\langle x, x^{\prime}\right\rangle \wedge\left\langle y, y^{\prime}\right\rangle=\left\langle y, y^{\prime}\right\rangle \wedge\left\langle x, x^{\prime}\right\rangle$

(d) $\left\langle x, x^{\prime}\right\rangle \preceq\left\langle y, y^{\prime}\right\rangle \Rightarrow\left\langle x, x^{\prime}\right\rangle \wedge\left\langle y, y^{\prime}\right\rangle=\left\langle x, x^{\prime}\right\rangle$

Proof. (a) From $\langle 1,0\rangle \vee\left\langle x, x^{\prime}\right\rangle=\langle 1,0\rangle$ we get $\left\langle x, x^{\prime}\right\rangle \preceq\langle 1,0\rangle, \Rightarrow\left\langle x, x^{\prime}\right\rangle \wedge\left\langle y, y^{\prime}\right\rangle \preceq\langle 1,0\rangle \wedge\left\langle y, y^{\prime}\right\rangle=\left\langle y, y^{\prime}\right\rangle$.

(b) From $\langle 1,0\rangle \vee\left\langle y, y^{\prime}\right\rangle=\langle 1,0\rangle$, we get $\left\langle y, y^{\prime}\right\rangle \preceq\langle 1,0\rangle$, so that $\left\langle x, x^{\prime}\right\rangle \wedge\left\langle y, y^{\prime}\right\rangle \preceq\left\langle x, x^{\prime}\right\rangle \wedge\langle 1,0\rangle=\left\langle x, x^{\prime}\right\rangle$.

(c)-(d) From (a), (b) and Proposition 1.

Obviously, if $\left\langle x, x^{\prime}\right\rangle \wedge\left\langle y, y^{\prime}\right\rangle \preceq\left\langle y, y^{\prime}\right\rangle$ for every $\left\langle x, x^{\prime}\right\rangle,\left\langle y, y^{\prime}\right\rangle \in X$, then $\langle x, x ;\rangle \wedge\langle 1,0\rangle \preceq\langle 1,0\rangle$, so that $\langle 1,0\rangle \vee\left\langle x, x^{\prime}\right\rangle=\langle 1,0\rangle$

Theorem 2. Let $\preceq$ be connected that is, $\left\langle x, x^{\prime}\right\rangle \preceq\left\langle y, y^{\prime}\right\rangle$ or $\left\langle y, y^{\prime}\right\rangle \preceq\left\langle x, x^{\prime}\right\rangle$ for every $\left\langle x, x^{\prime}\right\rangle,\left\langle y, y^{\prime}\right\rangle \in X, \wedge$ is idempotent, $\langle 1,0\rangle \vee\left\langle x, x^{\prime}\right\rangle=\langle 1,0\rangle$ for all $\left\langle x, x^{\prime}\right\rangle \in X$ and if $n \times n$ IFMs $Q$ and $S$ over $X$ are transitive and $Q \preceq S$, then $Q \stackrel{c}{-} S^{T}$ is transitive IFM, where $S^{T}$ is the transpose of $S$.

Proof. Let $Q=\left(\left\langle q_{i j}, q_{i j}^{\prime}\right\rangle\right)$ and $S=\left(\left\langle s_{i j}, s_{i j}^{\prime}\right\rangle\right)$, we have to prove that,

$$
\left(\left\langle q_{i k}, q_{i k}^{\prime}\right\rangle \stackrel{c}{\leftarrow}\left\langle s_{k i}, s_{k i}^{\prime}\right\rangle\right) \wedge\left(\left\langle q_{k j}, q_{k j}^{\prime}\right\rangle \stackrel{c}{\leftarrow}\left\langle s_{j k}, s_{j k}^{\prime}\right\rangle\right) \preceq\left(\left\langle q_{i j}, q_{i j}^{\prime}\right\rangle \stackrel{c}{\leftarrow}\left\langle s_{j i}, s_{j i}^{\prime}\right\rangle\right)
$$

Case (1): $\left\langle s_{j i}, s_{j i}^{\prime}\right\rangle \preceq\left\langle q_{k j}, q_{k j}^{\prime}\right\rangle \preceq\left\langle q_{i k}, q_{i k}^{\prime}\right\rangle$. Since $\left\langle q_{i k}, q_{i k}^{\prime}\right\rangle \preceq\left\langle s_{i k}, s_{i k}^{\prime}\right\rangle$ by Lemma 3 and transitivity of $\mathrm{S}$,

$$
\left\langle s_{j i}, s_{j i}^{\prime}\right\rangle=\left\langle s_{j i}, s_{j i}^{\prime}\right\rangle \wedge\left\langle s_{i k}, s_{i k}^{\prime}\right\rangle \preceq\left\langle s_{j k}, s_{j k}^{\prime}\right\rangle
$$

By transitivity of Q,

$$
\left\langle q_{i k}, q_{i k}^{\prime}\right\rangle \wedge\left\langle q_{k j}, q_{k j}^{\prime}\right\rangle \preceq\left\langle q_{i j}, q_{i j}^{\prime}\right\rangle,
$$


so that $\left\langle q_{k j}, q_{k j}^{\prime}\right\rangle \preceq\left\langle q_{i j}, q_{i j}^{\prime}\right\rangle$. By using Lemma 2, we get $\left(\left\langle q_{k j}, q_{k j}^{\prime}\right\rangle \stackrel{c}{\leftarrow}\left\langle s_{j k}, s_{j k}^{\prime}\right\rangle\right) \preceq\left(\left\langle q_{i j}, q_{i j}^{\prime}\right\rangle \stackrel{c}{\leftarrow}\left\langle s_{j i}, s_{j i}^{\prime}\right\rangle\right)$ By Lemma 3 we get (3).

Case (2): $\left\langle s_{j i}, s_{j i}^{\prime}\right\rangle \preceq\left\langle q_{i k}, q_{i k}^{\prime}\right\rangle \preceq\left\langle q_{k j}, q_{k j}^{\prime}\right\rangle$. Since $\left\langle q_{k j}, q_{k j}^{\prime}\right\rangle \preceq\left\langle s_{k j}, s_{k j}^{\prime}\right\rangle$

$$
\left\langle s_{j i}, s_{j i}^{\prime}\right\rangle=\left\langle s_{k j}, s_{k j}^{\prime}\right\rangle \wedge\left\langle s_{j i}, s_{j i}^{\prime}\right\rangle \preceq\left\langle s_{k i}, s_{k i}^{\prime}\right\rangle
$$

By (4) $\left\langle q_{i k}, q_{i k}^{\prime}\right\rangle \preceq\left\langle q_{i j}, q_{i j}^{\prime}\right\rangle$. Hence $\left(\left\langle q_{i k}, q_{i k}^{\prime}\right\rangle \stackrel{c}{\leftarrow}\left\langle s_{k i}, s_{k i}^{\prime}\right\rangle\right) \preceq\left(\left\langle q_{i j}, q_{i j}^{\prime}\right\rangle \stackrel{c}{\leftarrow}\left\langle s_{j i}, s_{j i}^{\prime}\right\rangle\right)$. Thus we get (3)

Case (3): $\left\langle q_{k j}, q_{k j}^{\prime}\right\rangle \preceq\left\langle s_{j i}, s_{j i}^{\prime}\right\rangle \preceq\left\langle q_{i k}, q_{i k}^{\prime}\right\rangle$ or $\left\langle q_{k j}, q_{k j}^{\prime}\right\rangle \preceq\left\langle q_{i k}, q_{i k}^{\prime}\right\rangle \preceq\left\langle s_{j i}, s_{j i}^{\prime}\right\rangle$, then

$$
\left\langle q_{k j}, q_{k j}^{\prime}\right\rangle \preceq\left(\left\langle s_{j i}, s_{j i}^{\prime}\right\rangle \wedge\left\langle s_{i k}, s_{i k}^{\prime}\right\rangle\right) \preceq\left\langle s_{j k}, s_{j k}^{\prime}\right\rangle
$$

$\Rightarrow\left(\left\langle q_{k j}, q_{k j}^{\prime}\right\rangle \stackrel{c}{\leftarrow}\left\langle s_{j k}, s_{j k}^{\prime}\right\rangle\right)=(\langle 0,1\rangle)$.

Case (4): $\left\langle q_{i k}, q_{i k}^{\prime}\right\rangle \preceq\left\langle s_{j i}, s_{j i}^{\prime}\right\rangle \preceq\left\langle q_{k j}, q_{k j}^{\prime}\right\rangle$ or $\left\langle q_{i k}, q_{i k}^{\prime}\right\rangle \preceq\left\langle q_{k j}, q_{k j}^{\prime}\right\rangle \preceq\left\langle s_{j i}, s_{j i}^{\prime}\right\rangle$. Then

$$
\left\langle q_{i k}, q_{i k}^{\prime}\right\rangle \preceq\left(\left\langle s_{k j}, s_{k j}^{\prime}\right\rangle \wedge\left\langle s_{j i}, s_{j i}^{\prime}\right\rangle\right) \preceq\left\langle s_{k i}, s_{k i}^{\prime}\right\rangle
$$

$\Rightarrow\left(\left\langle q_{i k}, q_{i k}^{\prime}\right\rangle \stackrel{c}{\leftarrow}\left\langle s_{k i}, s_{k i}^{\prime}\right\rangle\right)=(\langle 0,1\rangle)$

Obviously, by the conditions of Theorem $2 ; Q \stackrel{c}{\leftarrow} S^{T}$ is irreflexive, that is, all diagonal elements are $\langle 0,1\rangle$.

Example 3. Consider Q and S transitive IFMs

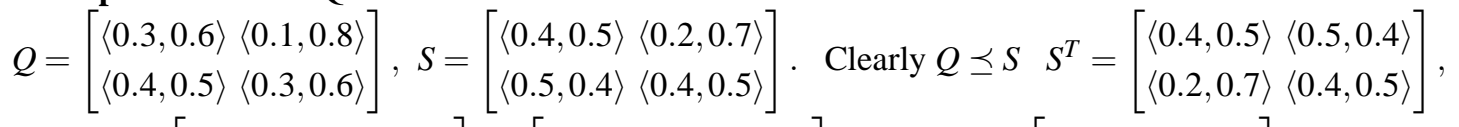

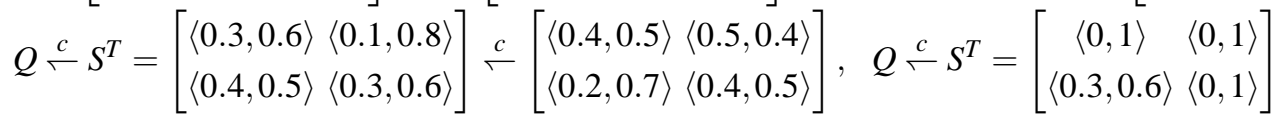

Therefore, $Q \stackrel{c}{\ulcorner} S^{T}$ is transitive IFM.

Example 4. We define

$$
\begin{aligned}
& \mathrm{X}=\left\{\left\langle x, x^{\prime}\right\rangle \mid\langle 0,1\rangle \leq\left\langle x, x^{\prime}\right\rangle \leq\langle 1,0\rangle\right\} \\
&\left\langle x, x^{\prime}\right\rangle-\left\langle y, y^{\prime}\right\rangle= \begin{cases}\left\langle x-y, y^{\prime}-x^{\prime}\right\rangle & \text { if }\left\langle x, x^{\prime}\right\rangle>\left\langle y, y^{\prime}\right\rangle, \\
\langle 0,1\rangle & \text { if }\left\langle x, x^{\prime}\right\rangle \leq\left\langle y, y^{\prime}\right\rangle\end{cases} \\
&\left\langle x, x^{\prime}\right\rangle \vee\left\langle y, y^{\prime}\right\rangle=\left\langle\max \{x, y\}, \min \left\{x^{\prime}, y^{\prime}\right\}\right\rangle \\
&\left\langle x, x^{\prime}\right\rangle \wedge\left\langle y, y^{\prime}\right\rangle=\left\langle\min \{x, y\}, \max \left\{x^{\prime}, y^{\prime}\right\}\right\rangle
\end{aligned}
$$

for every $\left\langle x, x^{\prime}\right\rangle,\left\langle y, y^{\prime}\right\rangle \in X$.That means above operation $\left\langle x, x^{\prime}\right\rangle-\left\langle y, y^{\prime}\right\rangle$ satisfies the conditions of Remark 1. An operation $\left\langle x, x^{\prime}\right\rangle-\left\langle y, y^{\prime}\right\rangle$ also satisfies the properties as below;

$$
\left\langle x, x^{\prime}\right\rangle-\left\langle y, y^{\prime}\right\rangle= \begin{cases}\left\langle x, x^{\prime}\right\rangle & \text { if }\left\langle x, x^{\prime}\right\rangle>\left\langle y, y^{\prime}\right\rangle, \\ \langle 0,1\rangle & \text { if }\left\langle x, x^{\prime}\right\rangle \leq\left\langle y, y^{\prime}\right\rangle .\end{cases}
$$

Proposition 2. If $\left\langle x, x^{\prime}\right\rangle \preceq\left\langle y, y^{\prime}\right\rangle$ implies $\left\langle x, x^{\prime}\right\rangle \wedge\left\langle y, y^{\prime}\right\rangle=\left\langle x, x^{\prime}\right\rangle$ for all $\left\langle x, x^{\prime}\right\rangle,\left\langle y, y^{\prime}\right\rangle \in X$ and if an $n \times n$ IFM $Q$ over $X$ is transitive and symmetric, that is $Q^{2} \preceq Q, Q^{T}=Q$, then $Q^{2}=Q$. 
Proof. Let an IFM $Q=\left\langle q_{i j}, q_{i j}^{\prime}\right\rangle$ over X be transitive and symmetric, we get $\left\langle q_{j i}, q_{j i}^{\prime}\right\rangle \wedge\left\langle q_{i j}, q_{i j}^{\prime}\right\rangle \preceq\left\langle q_{j j}, q_{j j}^{\prime}\right\rangle$, so that $\left\langle q_{i j}, q_{i j}^{\prime}\right\rangle \wedge\left\langle q_{i j}, q_{i j}^{\prime}\right\rangle \preceq\left\langle q_{j j}, q_{j j}^{\prime}\right\rangle$. From $\left\langle q_{i j}, q_{i j}^{\prime}\right\rangle \preceq\left\langle q_{i j}, q_{i j}^{\prime}\right\rangle$, we get $\left\langle q_{i j}, q_{i j}^{\prime}\right\rangle \preceq\left\langle q_{j j}, q_{j j}^{\prime}\right\rangle$. Then

$$
\begin{aligned}
& \bigvee_{k=1}^{n}\left(\left\langle q_{i k}, q_{i k}^{\prime}\right\rangle \wedge\left\langle q_{k j}, q_{k j}^{\prime}\right\rangle\right)=\left[\bigvee_{k=1, k \neq j}^{n}\left(\left\langle q_{i k}, q_{i k}^{\prime}\right\rangle \wedge\left\langle q_{k j}, q_{k j}^{\prime}\right\rangle\right)\right] \vee\left(\left\langle q_{i j}, q_{i j}^{\prime}\right\rangle \wedge\left\langle q_{j j}, q_{j j}\right\rangle\right) \\
\Rightarrow & \left.\bigvee_{k=1}^{n}\left(\left\langle q_{i k}, q_{i k}^{\prime}\right\rangle \wedge\left\langle q_{k j}, q_{k j}^{\prime}\right\rangle\right)=\left[\bigvee_{k=1, k \neq j}^{n}\left(\left\langle q_{i k}, q_{i k}^{\prime}\right\rangle \wedge\left\langle q_{k j}, q_{k j}^{\prime}\right\rangle\right)\right] \vee\left\langle q_{i j}, q_{i j}^{\prime}\right\rangle\right)
\end{aligned}
$$

Therefore, $\left\langle q_{i j}, q_{i j}^{\prime}\right\rangle \preceq \bigvee_{k=1}^{n}\left(\left\langle q_{i k}, q_{i k}^{\prime}\right\rangle \wedge\left\langle q_{k j}, q_{k j}^{\prime}\right\rangle\right), \Rightarrow Q \leq Q^{2}$, but we have $Q^{2} \leq Q$. Thus, $Q^{2}=Q$

Definition 10. An operation $\left\langle x, x^{\prime}\right\rangle \leftarrow\left\langle y, y^{\prime}\right\rangle \in X$ is defined as below: For every $\left\langle x, x^{\prime}\right\rangle,\left\langle y, y^{\prime}\right\rangle,\left\langle z, z^{\prime}\right\rangle \in X$,

(a) $\left\langle x, x^{\prime}\right\rangle \preceq\left\langle y, y^{\prime}\right\rangle$ then $\left\langle y, y^{\prime}\right\rangle \leftarrow\left\langle x, x^{\prime}\right\rangle=\langle 1,0\rangle$

(b) $\left(\left\langle x, x^{\prime}\right\rangle \leftarrow\left\langle y, y^{\prime}\right\rangle\right) \wedge\left(\left\langle y, y^{\prime}\right\rangle \leftarrow\left\langle z, z^{\prime}\right\rangle\right) \preceq\left\langle x, x^{\prime}\right\rangle \leftarrow\left\langle z, z^{\prime}\right\rangle$.

Example 5. Let $\mathrm{X}=\left\{\left\langle x, x^{\prime}\right\rangle \mid\langle 0,1\rangle \leq\left\langle x, x^{\prime}\right\rangle \leq\langle 1,0\rangle\right\}$,

$$
\begin{gathered}
\left\langle x, x^{\prime}\right\rangle \vee\left\langle y, y^{\prime}\right\rangle=\left\langle\max \{x, y\}, \min \left\{x^{\prime}, y^{\prime}\right\}\right\rangle \\
\left\langle x, x^{\prime}\right\rangle \wedge\left\langle y, y^{\prime}\right\rangle=\left\langle\min \{x, y\}, \max \left\{x^{\prime}, y^{\prime}\right\}\right\rangle \\
\left\langle x, x^{\prime}\right\rangle \leftarrow\left\langle y, y^{\prime}\right\rangle= \begin{cases}\langle 1,0\rangle & \text { if }\left\langle x, x^{\prime}\right\rangle \geq\left\langle y, y^{\prime}\right\rangle, \\
\left\langle x, x^{\prime}\right\rangle & \text { if }\left\langle x, x^{\prime}\right\rangle<\left\langle y, y^{\prime}\right\rangle .\end{cases}
\end{gathered}
$$

for every $\left\langle x, x^{\prime}\right\rangle,\left\langle y, y^{\prime}\right\rangle \in X$. Then $\left\langle x, x^{\prime}\right\rangle \preceq\left\langle y, y^{\prime}\right\rangle$ if and only if $\left\langle x, x^{\prime}\right\rangle \leq\left\langle y, y^{\prime}\right\rangle$,

$\left(\left\langle x, x^{\prime}\right\rangle \leftarrow\left\langle y, y^{\prime}\right\rangle\right) \wedge\left(\left\langle y, y^{\prime}\right\rangle \leftarrow\left\langle z, z^{\prime}\right\rangle\right) \preceq\left\langle x, x^{\prime}\right\rangle \leftarrow\left\langle z, z^{\prime}\right\rangle$.

Proposition 3. For every $\left\langle x, x^{\prime}\right\rangle,\left\langle y, y^{\prime}\right\rangle,\left\langle z, z^{\prime}\right\rangle \in X$, if $\left\langle x, x^{\prime}\right\rangle \preceq\left\langle y, y^{\prime}\right\rangle$, then $\left\langle z, z^{\prime}\right\rangle \leftarrow\left\langle y, y^{\prime}\right\rangle \preceq\left\langle z, z^{\prime}\right\rangle \stackrel{c}{\leftarrow}\left\langle x, x^{\prime}\right\rangle,\left\langle x, x^{\prime}\right\rangle \leftarrow\left\langle z, z^{\prime}\right\rangle \preceq\left\langle y, y^{\prime}\right\rangle \leftarrow\left\langle z, z^{\prime}\right\rangle$,

Proof. From definition $\left(\left\langle z, z^{\prime}\right\rangle \leftarrow\left\langle y, y^{\prime}\right\rangle\right) \wedge\left(\left\langle y, y^{\prime}\right\rangle \leftarrow\left\langle x, x^{\prime}\right\rangle\right) \preceq\left\langle z, z^{\prime}\right\rangle \leftarrow\left\langle x, x^{\prime}\right\rangle$.

Since $\left\langle x, x^{\prime}\right\rangle \preceq\left\langle y, y^{\prime}\right\rangle$, we have $\left\langle y, y^{\prime}\right\rangle \leftarrow\left\langle x, x^{\prime}\right\rangle=\langle 1,0\rangle$, so that $\left\langle z, z^{\prime}\right\rangle \leftarrow\left\langle y, y^{\prime}\right\rangle \preceq\left\langle z, z^{\prime}\right\rangle \leftarrow\left\langle x, x^{\prime}\right\rangle$,

In the same way $\left(\left\langle y, y^{\prime}\right\rangle \leftarrow\left\langle x, x^{\prime}\right\rangle\right) \wedge\left(\left\langle x, x^{\prime}\right\rangle \leftarrow\left\langle z, z^{\prime}\right\rangle\right) \preceq\left\langle y, y^{\prime}\right\rangle \leftarrow\left\langle z, z^{\prime}\right\rangle$, so that $\left\langle x, x^{\prime}\right\rangle \leftarrow\left\langle z, z^{\prime}\right\rangle \preceq\left\langle y, y^{\prime}\right\rangle \leftarrow\left\langle z, z^{\prime}\right\rangle$.

Lemma 4. For every $\left\langle x, x^{\prime}\right\rangle,\left\langle y, y^{\prime}\right\rangle,\left\langle u, u^{\prime}\right\rangle,\left\langle v, v^{\prime}\right\rangle \in X$, if $\left\langle u, u^{\prime}\right\rangle \preceq\left\langle y, y^{\prime}\right\rangle$, then

$$
\left(\left\langle x, x^{\prime}\right\rangle \leftarrow\left\langle y, y^{\prime}\right\rangle\right) \wedge\left(\left\langle u, u^{\prime}\right\rangle \leftarrow\left\langle v, v^{\prime}\right\rangle\right) \preceq\left\langle x, x^{\prime}\right\rangle \leftarrow\left\langle v, v^{\prime}\right\rangle .
$$

Proof. Let $\left\langle x, x^{\prime}\right\rangle \leftarrow\left\langle y, y^{\prime}\right\rangle \preceq\left\langle x, x^{\prime}\right\rangle \leftarrow\left\langle u, u^{\prime}\right\rangle$, we get

$$
\left(\left\langle x, x^{\prime}\right\rangle \leftarrow\left\langle y, y^{\prime}\right\rangle\right) \wedge\left(\left\langle u, u^{\prime}\right\rangle \leftarrow\left\langle v, v^{\prime}\right\rangle\right) \preceq\left(\left\langle x, x^{\prime}\right\rangle \leftarrow\left\langle u, u^{\prime}\right\rangle\right) \wedge\left(\left\langle u, u^{\prime}\right\rangle \leftarrow\left\langle v, v^{\prime}\right\rangle\right) \preceq\left\langle x, x^{\prime}\right\rangle \leftarrow\left\langle v, v^{\prime}\right\rangle .
$$

Theorem 3. Let $A$ and $B$ be $m \times n$ IFMs over $X$. If $A \preceq B$ and $\wedge$ is commutative, then $A \leftarrow B^{T}$ is transitive.

Proof. Let $A=\left(\left\langle a_{i j}, a_{i j}^{\prime}\right\rangle\right), B=\left[\left\langle b_{i j}, b_{i j}^{\prime}\right\rangle\right]$, and $Q=\left[\left\langle q_{i j}, q_{i j}^{\prime}\right\rangle\right]=A \leftarrow B^{T}$. Then

$$
\left\langle q_{i j}, q_{i j}^{\prime}\right\rangle=\bigwedge_{k=1}^{n}\left(\left(\left\langle a_{i k}, a_{i k}^{\prime}\right\rangle\right) \leftarrow\left(\left\langle b_{j k}, b_{j k}^{\prime}\right\rangle\right)\right) .
$$

By commutativity of $\wedge$

$$
\left\langle q_{i l}, q_{i l}^{\prime}\right\rangle \wedge\left\langle q_{l j}, q_{l j}^{\prime}\right\rangle=\bigwedge_{k=1}^{n}\left(\left(\left\langle a_{i k}, a_{i k}^{\prime}\right\rangle\right) \leftarrow\left(\left\langle b_{l k}, b_{l k}^{\prime}\right\rangle\right) \wedge\left(\left\langle a_{l k}, a_{l k}^{\prime}\right\rangle\right) \leftarrow\left(\left\langle b_{j k}, b_{j k}^{\prime}\right\rangle\right)\right)
$$


Using Lemma 4;

$$
\left.\left.\left(\left(\left\langle a_{i k}, a_{i k}^{\prime}\right\rangle\right) \leftarrow\left(b_{l k}, b_{l k}^{\prime}\right\rangle\right)\right) \wedge\left(\left(\left\langle a_{l k}, a_{l k}^{\prime}\right\rangle\right) \leftarrow\left(b_{j k}, b_{j k}^{\prime}\right\rangle\right)\right) \preceq\left\langle a_{i k}, a_{i k}^{\prime}\right\rangle \leftarrow\left\langle b_{j k}, b_{j k}^{\prime}\right\rangle
$$

Therefore

$$
\left\langle q_{i l}, q_{i l}^{\prime}\right\rangle \wedge\left\langle q_{l j}, q_{l j}^{\prime}\right\rangle \preceq\left\langle q_{i j}, q_{i j}^{\prime}\right\rangle .
$$

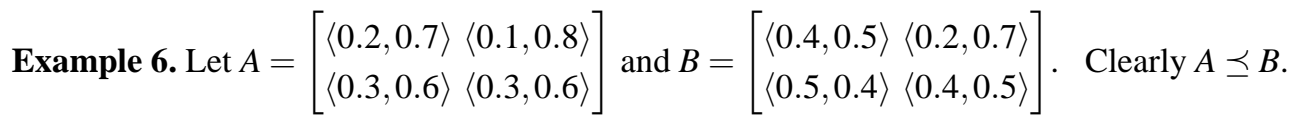

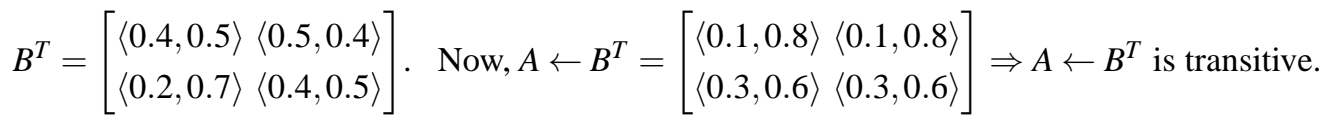

\section{Conclusion}

In this paper problem of transitivity of GIFMs over path algebra is discussed and some important properties are obtained.

\section{Acknowledgment}

The authors are very grateful and would like to express their sincere thanks to the anonymous referees and Editor-Saleem Abdulla for their valuable comments to improve the presentation of the paper.

\section{Competing interests}

The authors declare that they have no competing interests.

\section{Authors' contributions}

All authors have contributed to all parts of the article. All authors read and approved the final manuscript.

\section{References}

[1] L. A. Zadeh, Fuzzy Sets, Journal of Information and Control, 8, (1965), 338-353.

[2] K. Atanassov, Intuitionistic Fuzzy Sets, VII ITKR's Session, Sofia, June, 1983.

[3] K. Atanassov, Intuitionistic Fuzzy Sets, Fuzzy sets and systems, 20, (1986), 87-96.

[4] M. G Thomason, Convergence of powers of a fuzzy matrix, J. Math. Anal. Appl, 57, (1977), 476-480.

[5] K. H. Kim and F. W. Roush, Generalized fuzzy matrices, Fuzzy sets and systems, 4, (1980), 293-315.

[6] D. Dubois and H. Prade, Fuzzy Sets and Systems, vol. 144 of Mathematics in Science and Engineering, Academic Press, New York, NY, USA, 1980.

[7] J. A. Goguen, L-fuzzy sets Journal of Mathematical Analysis and Applications, 18 (1967) 145-174.

[8] A. Kaufmann, Introduction to the Theory of Fuzzy Subsets, Academic Press, New York, NY, USA, 1975.

[9] S. V. Ovchinnikov, Structure of fuzzy binary relations, Fuzzy Sets and Systems, 6(2), (1981), 169-195,

[10] L. A. Zadeh, Similarity relations and fuzzy orderings, Information Sciences, 3, (1971), 177-200.

[11] V. Tahani, A fuzzy model of document retrieval systems, Information Processing Management, 12, (1976), 177-187. 
[12] S. Tamura, S. Higuchi, and K. Tanaka, Pattern classification based on fuzzy relations, IEEE Transictions on Systems, Man, and Cybernetics, 1(1), (1971), 61-66.

[13] H. Hashimoto, Transitivity of generalized fuzzy matrices, Fuzzy Sets and Systems, 17(1), (1985), 83-90.

[14] W. Koodziejczyk, Convergence of powers of s-transitive fuzzy matrices, Fuzzy Sets and Systems, 26(1), (1988), 127-130.

[15] H. Hashimoto, Convergence of powers of a fuzzy transitive matrix, Fuzzy Sets and Systems, 9(2), (1983), 153-160.

[16] H. Hashimoto, Canonical form of a transitive fuzzy matrix, Fuzzy Sets and Systems, 11(2), (1983), 157-162.

[17] W. Koodziejczyk, Canonical form of a strongly transitive fuzzy matrix, Fuzzy Sets and Systems, 22(3), (1987), $297-302$.

[18] C. G. Hao, Canonical form of strongly transitive matrices over lattices, Fuzzy Sets and Systems, 45(2), (1992) 219-222.

[19] Y. J. Tan, On the powers of matrices over a distributive lattice, Linear Algebra and its Applications, 336, (2001), 1-14.

[20] Y. J. Tan, On the transitive matrices over distributive lattices, Linear Algebra and its Applications, 400, (2005), 169-191.

[21] L. J.-Xin, Controllable fuzzy matrices, Fuzzy Sets and Systems, 45, (1992), 313-319.

[22] L. J.-Xin, Convergence of powers of controllable fuzzy matrices, Fuzzy Sets and Systems, 62, (1994), 83-88.

[23] J. Jiang, L. Shu, and X. Tian, On generalized transitive matrices, Journal of Applied Mathematics, 2011 ID 164371, (2011) 1-16.

[24] Y.J. Tan, On transitivity of generalized fuzzy matrices, Fuzzy Sets and Systems, 210 (2013) 69-88

[25] M.Pal, Intuitionistic fuzzy determinant, V.U.J.Physical Sciences, 7, (2001), 87-93.

[26] M.Pal, S.Khan and A.K.Shyamal, Intuitionistic fuzzy matrices, Notes on Intuitionistic Fuzzy Sets, 8 (2), (2002), 51-62.

[27] S.K.Khan and M.Pal, Intuitionistic fuzzy tautological matrices, V.U. Journal of Physical Sciences , 8, (2002-2003), 92-100.

[28] S.K.Khan and M.Pal, Interval valued intuitionistic fuzzy matrices, Communicated.

[29] M. Bhowmik , M. Pal , Some results on intuitionistic fuzzy matrices and intuitionistic circulant fuzzy matrices. International Journal of Mathematical Sciences 7(1-2): (2008), 177-192

[30] M. Bhowmik, M. Pal, Generalized intuitionistic fuzzy matrices. Far-East Journal of Mathematical Sciences 29(3), (2008) 533-554.

[31] S. K. Khan and A. Pal, The generalized inverse of intuitionistic fuzzy matrices, Journal of Physical Sciences, 11, $2007,62-67$.

[32] H. Y. Lee and N. G. Jeong. Canonical form of a transitive intuitionistic fuzzy matrices, Honam Mathematical Journal, 27(4), (2005) 543-550.

[33] A. K. Adak, M. Bhowmik and M. Pal, Some properties of generalized intuitionistic fuzzy nilpotent matrices over distributive lattice, Fuzzy Inf. Eng. 4, (2012), 371-387.

[34] R. Pradhan and M. Pal, Some results on generalized Inverse of intuitionistic fuzzy matrices, Fuzzy Inf. Eng. 6, (2014), $133-145$.

[35] A. R.Meenakshi, and T. Gandhimathi, Intuitionistic fuzzy relational equations, advances in Fuzzy Mathematics, 5, (3), (2010), 239-244.

[36] S. Mondal and M. Pal, Similarity relations, invertibility and eigenvalues of intuitionistic fuzzy matrix, International Journal of Fuzzy Information and Engineering, 4, (2013), 431-443.

[37] S. Mondal and M. Pal, Intuitionistic fuzzy incline matrix and determinant, Annals of Fuzzy Mathematics and Informatics, 8(1), (2014), 19-32.

[38] P. Murugadas and K. Lalitha, Sub-inverse and g-inverse of an Intuitionistic Fuzzy Matrix Using Bi-implication Operator, Int.Journal of Computer Application. 89, (1), (2014), 1-5.

[39] R. Pradhan and M. Pal, Convergence of maxarithmetic mean-minarithmetic mean powers of intuitionistic fuzzy matrices, intern. J. Fuzzy Mathematical Archive, 2, (2013) 58-69.

[40] R. Pradhan and M. Pal, Intuitionistic fuzzy linear transformations, Annals of pure and applied Mathematics, 1(1), (2012), 57-68.

[41] R. Pradhan and M. Pal, The generalized inverse of Atanassov's intuitionistic fuzzy matrices, International Journal of Computational Intelligence Systems, 7(6), (2014), 1083-1095.

[42] S. Sriram and P. Murugadas, On semi-ring of intuitionistic fuzzy matrices, Applied Mathematical Science, 4(23), (2010), 10991105.

[43] S. Sriram and P. Murugadas, Sub-inverses of intuitionistic fuzzy matrices, Acta Ciencia Indica Mathematics, Vol.XXXVII, M No.1, (2011), 41-56.

[44] A. K. Shyamal and M. Pal, Distance between intuitionistic fuzzy matrices, V.U.J. Physical Sciences, 8, (2002), 81-91

[45] R. A. Padder and P. Murugadas, Max-max operation on intuitionistic fuzzy matrix, Annals of Fuzzy Mathematics and Informatics, Article in press. 
[46] P.Murugadas and R. A. Padder, Reduction of an intuitionistic fuzzy rectangular Matrix, Annamalai University Science Journal, 49 , (2015), 15-18.

[47] R. A. Padder and P. Murugadas, Convergence of Power of Controllable Intuitionistic Fuzzy Matrices, ICTACT Journal on Soft Computing 07(01), (2016), 1332-1337.

[48] R. A. Padder and P. Murugadas, On Idempotent Intuitionistic Fuzzy Matrices of T-type, International Journal of Fuzzy Logic and Intelligent Systems, 16(3), (2016), 181-187. 